\title{
Quantum many-body scars in spin-1 Kitaev chains
}

\author{
Wen-Long You $\odot,{ }^{1,2}$ Zhuan Zhao, ${ }^{1,2}$ Jie Ren $\odot,{ }^{3}$ Gaoyong Sun $\odot,{ }^{1,2}$ Liangsheng Li $\odot,{ }^{4}$ and Andrzej M. Oleś $\odot^{5,6, *}$ \\ ${ }^{1}$ College of Science, Nanjing University of Aeronautics and Astronautics, Nanjing 211106, China \\ ${ }^{2}$ Key Laboratory of Aerospace Information Materials and Physics (NUAA), MIIT, Nanjing 211106, China \\ ${ }^{3}$ Department of Physics, Changshu Institute of Technology, Changshu 215500, China \\ ${ }^{4}$ Science and Technology on Electromagnetic Scattering Laboratory, Beijing 100854, China \\ ${ }^{5}$ Max Planck Institute for Solid State Research, Heisenbergstrasse 1, D-70569 Stuttgart, Germany \\ ${ }^{6}$ Institute of Theoretical Physics, Jagiellonian University, Prof. Stanisława Łojasiewicza 11, PL-30348 Kraków, Poland
}

(Received 19 October 2021; accepted 20 January 2022; published 10 February 2022)

\begin{abstract}
To provide a physical example of quantum scars, we study the many-body scars in the spin-1 Kitaev chain where the so-called PXP Hamiltonian is exactly embedded in the spectra. Regarding the conserved quantities, the Hilbert space is fragmented into disconnected subspaces and we explore the associated constrained dynamics. The continuous revivals of the fidelity and the entanglement entropy when the initial state is prepared in $\left|\mathbb{Z}_{k}\right\rangle(k=2,3)$ state illustrate the essential physics of the PXP model. We study the quantum phase transitions in the one-dimensional spin-1 Kitaev-Heisenberg model using the density-matrix renormalization group and Lanczos exact diagonalization methods, and determine the phase diagram. We parametrize the two terms in the Hamiltonian by the angle $\phi$, where the Kitaev term is $K \equiv \sin (\phi)$ and competes with the Heisenberg $J \equiv \cos (\phi)$ term. One finds a rich ground-state phase diagram as a function of the angle $\phi$. Depending on the ratio $K / J \equiv \tan (\phi)$, the system either breaks the symmetry to one of distinct symmetry broken phases, or preserves the symmetry in a quantum spin-liquid phase with frustrated interactions. We find that the scarred state is stable for perturbations which obey $\mathbb{Z}_{2}$-symmetry, while it becomes unstable against Heisenberg-type perturbations.
\end{abstract}

DOI: 10.1103/PhysRevResearch.4.013103

\section{INTRODUCTION}

With the rapid development of experimental techniques, specifically with the advancement of stable and precisely adjustable ultrashort pulse laser technologies, and the improvement of detection methods, the real-time information with atomic scale and subpicosecond resolution can be obtained. It provides new opportunities for perceiving the phenomena in related many-body systems. Meanwhile, quantum simulators with high isolation, long relaxation time, and experimental controllability, such as ultracold atoms, ion traps, nitrogen vacancy centers in diamonds, and superconducting circuits, have opened up a new direction in the studies of strongly correlated electron systems in condensed matter physics. Studies of abstract quantum complex systems are another route to provide a more complete description of quantum phase transitions and many-body dynamics in isolated quantum systems.

Particularly in recent years, tremendous progress was made in describing the nonequilibrium dynamics of isolated

\footnotetext{
*a.m.oles@fkf.mpi.de

Published by the American Physical Society under the terms of the Creative Commons Attribution 4.0 International license. Further distribution of this work must maintain attribution to the author(s) and the published article's title, journal citation, and DOI. Open access publication funded by the Max Planck Society.
}

quantum systems. Thermalization and localization are distinct fates of the steady quantum states. The first will lose information of the initial state after undergoing a long-time evolution, while the second retains information of the initial state as much as possible. In general, the system will tend to thermalize as it follows the eigenstate thermalization hypothesis $(\mathrm{ETH})$, which is generally regarded as the cornerstone of contemporary quantum statistics. A system that satisfies ETH is considered to be ergodic. Numerical calculations show that most interacting systems are indeed strongly ergodic [1-4]. Thermal excitations enrich the properties of spin chains and are, for instance, responsible for the dimerization in spinorbital systems [5].

However, currently many quantum systems were found to disobey the ETH. A typical exception is the integrable system undergoing Anderson localization [6], in which there are infinite local conserved quantities that can inhibit the relaxation between eigenstates and thus retain the coherence in the quantum system. However, even a weak perturbation will suffice to break the integrability of the system, and then the ETH would be restored [7].

Another mechanism to avoid thermalization is manybody localization-it has been intensely investigated in the past decade [8-12]. The emergent integrability of these $a$ priori nonintegrable systems prevents them from thermalization [13]. The Anderson localization generally concerns the ground state, while the many-body localization usually occurs at finite temperature. Recently, the phase transitions between the thermalized and many-body localized phases, highlighting 
many-body localized mobility edge, were observed in 19qubit superconducting systems [14].

Nowadays exact quantum many-body scars (QMBSs) and their stability in constrained quantum chains attract attention [15]. Quantum scars are nonthermal eigenstates characterized by low entanglement entropy, initially detected in systems subject to nearest-neighbor Rydberg blockade, the PXP model [16-19]. This model has been introduced as a simplified description of the quantum simulator on 51 Rydberg atoms [20]. It has been shown that the quantum quench dynamics starting from an antiferromagnetic (AFM) state will return to the certain initial state repeatedly after reaching equilibrium. The continuous oscillations of physical observables such as domain-wall density show that the system exhibits a new type of nonthermalization behavior and the peculiar behavior was dubbed as QMBSs. If the initial state is prepared in other generic states, the atomic system will thermalize as expected. In addition to the Rydberg atomic system, QMBSs were also observed in one-dimensional (1D) dipolar Bose gas [21].

The ideas of the quantum scar state were borrowed from the classic chaotic scar phenomenon, which is characterized by a high probability density distribution near a specific classical periodic orbit. Quantum scars were originally proposed by Heller in 1984 based on the single-particle picture [22]. QMBSs are abnormal condensations of quantum many-body wave functions on specific eigenstates, and these eigenstates constitute quantum scars. On one hand, because QMBSs are different from the conventional paradigm of thermalization, Anderson localization, and many-body localization, the nature of this novel quantum phenomenon has stimulated the interest of many physicists. On the other hand, QMBSs may represent a new way to realize coherent quantum mechanics, which can protect the quantum dense coding processing for a long time. Obviously, searching for nonthermal dynamics in interacting nonintegrable quantum systems has not only fundamental theoretical significance in condensed matter theory, but also has great practical significance in quantum information processing.

In a nutshell, the scarred model is characterized by a subspace which is decoupled from the rest of the energy spectrum and cannot be simply attributed to a symmetry of the system. Taking the spin- $S$ system on a chain with length $N$ as an example, the dimension of the full Hilbert space scales exponentially as $(2 S+1)^{N}$, and the dimension of the scar subspace scales algebraically with the system size $N$ as $\mathcal{O}(N)$. These zero-measure scar eigenstates allow the quantum system to maintain long-time coherent dynamics. The underlying mechanism for the 51 Rydberg atoms finding the return journey of a specific initial state in the Hilbert space of more than $4 \times 10^{10}$ dimensions is fuzzy.

Ergodicity breaking in such systems can often be attributed to the presence of symmetries (hidden, emergent, or explicit) that preclude the establishment of a global equilibrium state $[23,24]$. The existence of quantum scars may be revelent to the emergent integrability of the Hamiltonian [25], the algebraic structure of dynamic symmetry [26], while some researches rule out the integrability [27].

A flurry of subsequent theoretical works addressed the puzzle posed by the experiment: the nature and origin of this new regime of ergodicity breaking, intermediate between ther- malization and strong ergodicity breaking. Notably, extensive research on weak ergodicity breaking was performed in various quantum many-body systems, such as the extended Ising model [28], integer spin $X Y$ model [29], Affleck-KennedyLieb-Tasaki (AKLT) model [30-32], and the Hubbard model [33-35]. It was demonstrated that using 1D so-called PXP models for spin-1 can lead to weak ergodicity breaking [12].

Kitaev models attracted much attention currently as they provide a deeper understanding of the spin-orbital physics in transition metal oxides [36]. The search for their phase diagrams is motivated by a topological quantum spin liquid (QSL) ground state and Majorana excitations emerging from the frustrated Kitaev model [37]. This research developed rapidly after a seminal paper of Jackeli and Khaliullin [38] was published. They proposed that the QSL might be realized in Mott insulators with strong spin-orbit coupling, opening a new route to seek for the Kitaev QSLs.

In recent years it was recognized that the $S=1$ variant could be designed by considering strong Hund's coupling among two electrons in $e_{g}$ orbitals and strong spin-orbit coupling at anion sites [39], and this innovative concept sparked intensive theoretical studies. The 1D version of the Kitaev model can be considered as a limiting case with vanishing interactions along $z$ bonds of the hexagonal lattice. The $X X$ and $Y Y$-type nearest-neighbor Ising interactions toggle sequentially between odd and even bonds in the 1D Kitaev chain. Despite severe simplification, the Kitaev chain shares some features of the honeycomb model.

In this paper, we study the spin-1 Kitaev chain, which is nonintegrable. We show that this model after increasing spin to $S=1$ surprisingly harbors an extensive set of anomalous scarred eigenstates at finite energy density that exhibit subextensive entanglement entropy. These scarred states survive certain Heisenberg perturbation. Our results thus firmly establish the existence of QMBSs in the 1D spin-1 Kitaev model. The entanglement entropy of a subregion $A$ in an eigenstate $\alpha$ is, $\mathcal{S}_{A}^{\alpha}=-\operatorname{Tr}\left\{\rho_{A}^{\alpha} \ln \rho_{A}^{\alpha}\right\}$, and ETH-obeying states have extensive volume-law entanglement entropy, $\mathcal{S}_{A}^{\alpha} \propto V$. Thus, to show that the quantum states violate the ETH, we need only to show that their entanglement entropy is subextensive.

The purpose of this paper is twofold. First, we would like to provide evidence that scarred eigenstates indeed appear naturally in the spin-1 Kitaev model as the PXP model is embedded in one of subspaces. Second, quantum scars in the many-body Hamiltonian relevant for electronic systems are also of interest. Thereby we are guided by the idea that studying a wide variety of interacting systems with exact scar states and their stability to perturbations would be beneficial for general understanding.

\section{SPIN-1 KITAEV CHAIN}

In the present paper, we focus on a spin-1 Kitaev chain

$$
\hat{H}_{K}=K \sum_{j=1}^{N / 2}\left(S_{2 j-1}^{x} S_{2 j}^{x}+S_{2 j}^{y} S_{2 j+1}^{y}\right),
$$

where spin operators $\left\{S_{j}^{a}\right\}$ (with $a=\{x, y, z\}$ ) are the spin-1 operators at site $j$, for a chain of $N$ sites. The spin operators 
obey the $\mathrm{SU}(2)$ algebra, $\left[S_{i}^{a}, S_{j}^{b}\right]=i \delta_{i j} \epsilon_{a b c} S_{j}^{c}$, with the totally antisymmetric tensor $\epsilon_{a b c}$ and $\left(\mathbf{S}_{j}\right)^{2}=S(S+1)=2$. The spin-1 Kitaev model in Eq. (1) breaks the global spin rotation $\mathrm{SU}(2)$ symmetry, while still obeying the time reversal symme$\operatorname{try} \mathcal{T},\left(S_{j}^{x, y, z} \rightarrow-S_{j}^{x, y, z}\right)$ and the spatial inversion symmetry $\mathcal{I}$, $\left(j \rightarrow N-j+1, S_{j}^{x, y, z} \rightarrow S_{N+1-j}^{x, y, z}\right)$. The sign of $K$ in Eq. (1) is irrelevant as far as a rotation $U_{z}=\prod_{j} \Sigma_{2 j}^{z}$ at each even lattice site by an angle $\pi$ about the $z$-axis will reverse the sign.

While the sign of the Kitaev interactions is still under debate, with conflicting results from theoretical and experimental studies, $\mathrm{RuCl}_{3}$ was deemed as a promising candidate for the Kitaev QSL with spin $S=\frac{1}{2}$ [40]. For the present model Eq. (1) we shall investigate whether QSL is also stable in a certain regime of parameters. Previous neutron scattering studies performed to complement density functional theory (DFT) suggested that the Kitaev interaction may be ferromagnetic (FM) or AFM. A recent experiment on $\alpha-\mathrm{RuCl}_{3}$ by measuring azimuthal dependence [41] suggests that the Kitaev interaction is here FM.

It is convenient to use a representation for $S=1$ given by the set of orthonormal states $\{|-\rangle,|0\rangle,|+\rangle\}$, where

$$
\begin{aligned}
& |-\rangle \equiv \frac{1}{\sqrt{2}}(|-1\rangle-|1\rangle), \\
& |+\rangle \equiv \frac{i}{\sqrt{2}}(|-1\rangle+|1\rangle),
\end{aligned}
$$

and $|m\rangle$ is an eigenstate of the spin operator $S_{i}^{z}$ to an eigenvalue $m=-1,0,1$, i.e., it stands for the state $|1, m\rangle$. Furthermore, spin-1 operators can be written as $S_{b c}^{a}=i \epsilon_{a b c}$. Explicitly, spin operators $\left\{S^{x}, S^{y}, S^{z}\right\}$ for spin $S=1$ may be represented by the matrices

$$
\left(\begin{array}{ccc}
0 & 0 & 0 \\
0 & 0 & -i \\
0 & i & 0
\end{array}\right), \quad\left(\begin{array}{ccc}
0 & 0 & i \\
0 & 0 & 0 \\
-i & 0 & 0
\end{array}\right), \quad\left(\begin{array}{ccc}
0 & -i & 0 \\
i & 0 & 0 \\
0 & 0 & 0
\end{array}\right) .
$$

The spin components define site parity operators $\Sigma_{j}^{a}=e^{i \pi S_{j}^{a}}$, i.e., $\left\{\Sigma^{x}, \Sigma^{y}, \Sigma^{z}\right\}$ are given by the matrices

$$
\left(\begin{array}{ccc}
1 & 0 & 0 \\
0 & -1 & 0 \\
0 & 0 & -1
\end{array}\right),\left(\begin{array}{ccc}
-1 & 0 & 0 \\
0 & 1 & 0 \\
0 & 0 & -1
\end{array}\right),\left(\begin{array}{ccc}
-1 & 0 & 0 \\
0 & -1 & 0 \\
0 & 0 & 1
\end{array}\right) .
$$

From the form of these operators we see that the eigenvalues of operators $\Sigma_{j}^{a}$ are \pm 1 , but -1 is doubly degenerate. The Hamiltonian in Eq. (1) respects the dihedral group $D_{2}$ for a global discrete symmetry with a rotation by an angle $\pi$ about the $\{x, y, z\}$ axes, i.e., $\prod_{j} \Sigma_{j}^{a}$. One finds that all $\Sigma_{j}^{a}$ matrices commute with each other. In addition, $\Sigma_{j}^{a}$ commutes with $S_{j}^{a}$ but anticommutes with $S_{j}^{b}(a \neq b)$, i.e., $\left\{\Sigma_{j}^{a}, S_{j}^{b}\right\}=\left\{\exp \left(i \pi S_{j}^{a}\right), S_{j}^{b}\right\}=0$. that

In terms of the ladder operators $S_{j}^{ \pm} \equiv S_{j}^{x} \pm i S_{j}^{y}$, one finds

$$
\begin{aligned}
& {\left[S_{j}^{+}, S_{j}^{-}\right]=2 S_{j}^{z}, \quad\left[S_{j}^{z}, S_{j}^{ \pm}\right]= \pm S_{j}^{ \pm},} \\
& \left\{S_{j}^{+}, S_{j}^{-}\right\}=2\left[2-\left(S_{j}^{z}\right)^{2}\right] .
\end{aligned}
$$

The Ising terms in Eq. (1) change the total pseudospin- $z$ at both $x$-link $(2 j-1,2 j)$ and $y$-link $(2 j, 2 j+1)$ by either 0 or \pm 2 . Thus the bond parity operators on odd/even bonds

$$
\hat{W}_{2 j-1}=\Sigma_{2 j-1}^{y} \Sigma_{2 j}^{y}, \text { and } \hat{W}_{2 j}=\Sigma_{2 j}^{x} \Sigma_{2 j+1}^{x},
$$

define the invariants of the Hamiltonian in Eq. (1). The different forms on odd and even bonds in Eq. (6) can be cured by a unitary transformation on the even sites

$$
S_{2 j}^{x} \rightarrow S_{2 j}^{y}, S_{2 j}^{y} \rightarrow S_{2 j}^{x}, \text { and } S_{2 j}^{z} \rightarrow-S_{2 j}^{z} .
$$

In the transformed frame, the Hamiltonian in Eq. (1) then takes a translationally invariant form

$$
\tilde{H}_{\mathrm{K}}=K \sum_{j=1}^{N} S_{j}^{x} S_{j+1}^{y},
$$

and the bond parity operators on odd/even bonds then simultaneously take a universally convenient form

$$
\tilde{W}_{j}=\Sigma_{j}^{y} \Sigma_{j+1}^{x} \text {. }
$$

The eigenvalues of $\tilde{W}_{j}$ are $w_{j}= \pm 1$. One observes from Eq. (4) that $\Sigma_{j}^{z}=\Sigma_{j}^{x} \Sigma_{j}^{y}$, implying two of them are independent, e.g., $\Sigma_{j}^{x}$ and $\Sigma_{j}^{y}$. The $\mathbb{Z}_{2}$-valued conserved quantity $\tilde{W}_{j}$ implies that the Hilbert space can be decomposed into $2^{N}$ sectors of unequal sizes for the spin-1 chain.

The projector at a subspace with a given set of invariants, $\left\{w_{1}, w_{2}, \ldots, w_{N}\right\}$, can be constructed as $\hat{Q}\left(w_{1}, w_{2}, \ldots, w_{N}\right)=\prod_{j}\left(1+w_{j} \tilde{W}_{j}\right) / 2$. It acts on a random state $\left|\psi_{\text {rand }}\right\rangle$ and the dimension $\mathcal{D}$ can be calculated by using the technique of the transfer matrix. The ground state of $\tilde{H}_{\mathrm{K}}$ with periodic boundary conditions (PBCs) lies in the sector with defect-free subspace [42]. Different from the spin-1/2 counterpart, where the uniform subspace, with parity being either $w_{j}=+1$ or $w_{j}=-1$ for all $j$, are all equivalent up to a unitary transformation, but the dimensions of these sectors are nonidentical. The dimension of the sector with all $w_{j}=-1$ is equal to $\mathcal{D}(\{-1,-1, \ldots,-1\})=2$, which appears to be the smallest subspace, as is evidenced in Fig. 1.

The two-dimensional subspace with all $w_{j}=-1$ can be rewritten as $|---\cdots--\rangle$ and $|+++\cdots++\rangle$ defined in the rotated bases in Eq. (7). On the contrary, the dimension $\mathcal{D}$ for the subspace with parity $w_{j}=+1$ for all $j$ is proven to be the Lucas number, i.e., $\mathcal{D}(\{1,1, \ldots, 1\})=F_{N-1}+F_{N+1}$, where $F_{n}$ is the $n$th Fibonacci number. More precisely, $\mathcal{D}(\{1,1, \ldots, 1\})=g^{N}+g^{-N}$, where $g=(1+\sqrt{5}) / 2 \approx 1.618$ is the golden ratio. It is interesting to observe that such fractal dimension-based subspace is the largest among all the sectors, see Fig. 1.

The constrained Hilbert space without any adjacent spin-up atoms makes the system identical to that of chains of spin$1 / 2$ atoms. Hence, the Hilbert space of a given sector can be mapped into the Hilbert space of a spin-1/2 chain with some states excluded. Accepting the notation that the $|\uparrow\rangle$ state represents the state $|-\rangle$ (head), and the $|\downarrow\rangle$ state stands for either the state $|0\rangle$ (empty) or the state $|+\rangle$ (tail), the system can be mapped to a single qubit-flip model with nearestneighbor exclusion represented by the effective Hamiltonian 


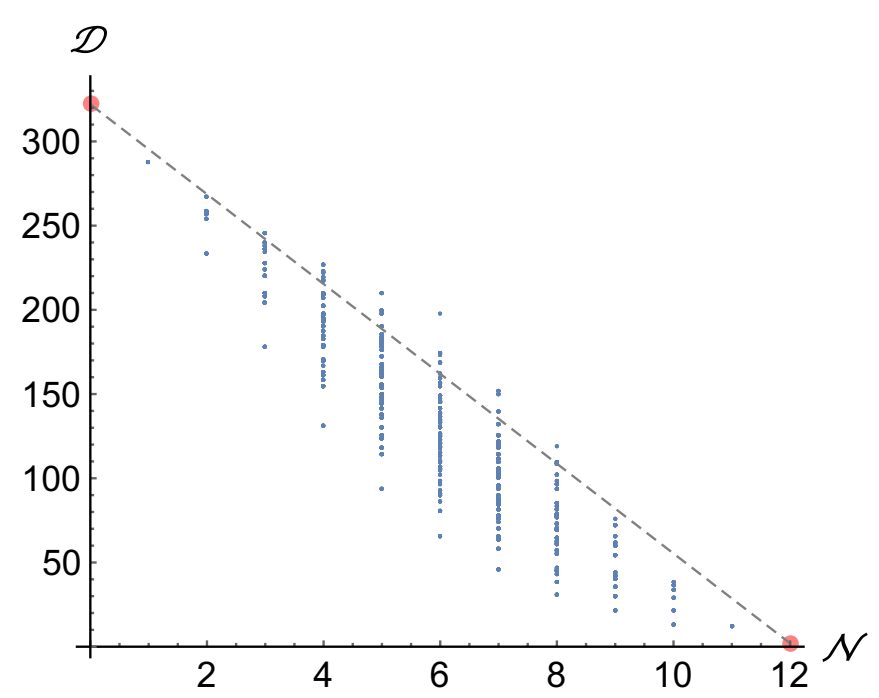

FIG. 1. The dimension of subspaces $\mathcal{D}$ for given parity $\left(w_{1}, w_{2}, \ldots, w_{N}\right)$ as a function of the defect number $\mathcal{N}=N-\sum_{j=1}^{N} w_{j}$, for a chain with $N=12$ sites. The red dot denotes the maximum (minimum) of $\mathcal{D}$ corresponding to the uniform subspace with parity $w_{j}=+1\left(w_{j}=-1\right)$ for all $j$. The dashed line is a guide for the eye.

in the sector with $w_{j}=+1$ for all $j$ [43]:

$$
\mathcal{H}_{\{1,1, \ldots, 1\}}=\sum_{j=1}^{N} \tilde{X}_{j} .
$$

Here $\quad \tilde{X}_{j} \equiv P_{j-1} X_{j} P_{j+1}$, where the Pauli operators $X_{j}=(|\uparrow\rangle\langle\downarrow|+| \downarrow\rangle\langle\uparrow|), \quad Z_{j}=(\mid(\uparrow\rangle\langle\uparrow|-| \downarrow\rangle\langle\downarrow|), \quad$ and the projectors $P_{j}=|\downarrow\rangle\langle\downarrow|=\left(1-Z_{j}\right) / 2$, ensure that the nearby atoms are not simultaneously in the excited state. The corresponding dimension of the constrained Hilbert space is exactly equal to $\mathcal{D}(\{1,1, \ldots, 1\})$ through an isomorphic mapping. Note that the PXP model is an effective model of the biaxial Ising model with both transverse and longitudinal fields, where the low field $h_{x} \ll J_{z}$ and the saturation field $h_{z}=2 J_{z}$ are present. In spite of its rather friendly form, the Hamiltonian Eq. (10) is nonintegrable due to the low-energy constraint imposed on the Hilbert space.

It was noted that the PXP model specified by the Hamiltonian (10) will exhibit QMBSs, which can be experimentally prepared and probed using a global quench. Experiment [20] and numerical simulations on small systems [44] revealed that the relaxation under unitary dynamics strongly depended on the initial state of the system. It was found that special eigenstates have anomalously high overlaps with certain product states.

The polarized state with all spins in $|\downarrow\rangle$ state $|\varnothing\rangle=\prod_{j} P_{j}\left|\psi_{\text {rand }}\right\rangle$ satisfies $\mathcal{H}_{\{1,1, \ldots, 1\}}|\varnothing\rangle=0$, which allows one to identify a dominant subset of special states in the PXP model. We initialize the system at time $t=0$ in the state $|\psi(0)\rangle \equiv\left|\mathbb{Z}_{k}\right\rangle$, namely,

$$
\left|\mathbb{Z}_{k}\right\rangle=\ldots \tilde{X}_{k} \ldots \tilde{X}_{2 k} \ldots|\varnothing\rangle
$$

and then follow the evolution of the initial state with the PXP Hamiltonian, $|\psi(t)\rangle=\exp \left(-i \mathcal{H}_{\{1,1, \ldots, 1\}} t\right)|\psi(0)\rangle$. The evolu- tion is determined by the decomposition of $|\psi(0)\rangle$ in terms of the eigenstates of $\mathcal{H}_{\{1,1, \ldots, 1\}}$. The initial states with atoms in all spin-down state $\left|\mathbb{Z}_{1}\right\rangle \equiv|\varnothing\rangle$ or $\left|\mathbb{Z}_{4}\right\rangle$ show fast relaxation and no revivals, characteristic of thermalizing systems. Remarkably, the quantum quench from either period-2 state $\left(\left|\mathbb{Z}_{2}\right\rangle\right)$ or the period-3 state $\left(\left|\mathbb{Z}_{3}\right\rangle\right)$ will surprisingly give rise to coherent oscillations, which can be observed in the dynamics by measuring the expectation values of certain local observables and the quantum fidelity

$$
F(t)=\left|\left\langle\mathbb{Z}_{k}|\exp (-i H t)| \mathbb{Z}_{k}\right\rangle\right| .
$$

The observed oscillations and the apparent nonergodic dynamics are attributed to the existence of QMBSs [45].

Intuitively, the empty state $|\varnothing\rangle$ corresponds to the state $|0000 \ldots 00\rangle$, which can be inferred from Eq. (4) that the configuration $|0000 \ldots 00\rangle$ resides in this subspace with uniform $\mathbb{Z}_{2}$ invariants, i.e., $w_{j}=1$ for all $j$. In this context, the akin states $\left|\tilde{\mathbb{Z}}_{k}\right\rangle$ of spin-1 Kitaev model Eq. (8) as product states in Eq. (11), can be reconciled by acting

$$
\left|\tilde{\mathbb{Z}}_{k}\right\rangle=\ldots S_{k}^{x} S_{k+1}^{y} \ldots S_{2 k}^{x} S_{2 k+1}^{y} \ldots|0000 \cdots 00\rangle .
$$

Then the corresponding spin-1 states are given by

$$
\begin{gathered}
\left|\tilde{\mathbb{Z}}_{2}\right\rangle=|-+-+\cdots-+\rangle, \\
\left|\tilde{\mathbb{Z}}_{3}\right\rangle=|+-0+-0 \cdots+-0\rangle, \\
\left|\tilde{\mathbb{Z}}_{4}\right\rangle=|+-00+-00 \cdots+-00\rangle .
\end{gathered}
$$

It is clear that the translated states, $\left|\tilde{\mathbb{Z}}_{k}^{\prime}\right\rangle=\left(T_{i \rightarrow i+1}\right)^{j}\left|\mathbb{Z}_{k}\right\rangle$ $(j=1,2, \ldots, k-1)$, also belong to the QMBSs, where $T_{i \rightarrow i+1}$ denotes the translation by one lattice site, i.e., $i \rightarrow i+1$. As is shown in Fig. 2(a), the revivals of the quantum fidelity for spin-1 Kitaev model (8) [spin-1/2 PXP model (10)] starting from the initial Néel state $\left|\tilde{\mathbb{Z}}_{2}\right\rangle\left(\left|\mathbb{Z}_{2}\right\rangle\right)$ are indistinguishable, suggesting the existence of ETH-violating QMBSs.

Figure 3 shows that the fidelity $F$ and entanglement $\mathcal{S}$ with its dynamics for different initial states are interrelated in a subtle way. One can observe the coherent oscillations of fidelity that persist for long times for quenches from initial product states $\left|\tilde{\mathbb{Z}}_{2}\right\rangle$ and $\left|\tilde{\mathbb{Z}}_{3}\right\rangle$ [18], with different revival periods, and the entanglement entropy $\mathcal{S}$, see Fig. 3(b), is gradually growing with time. The weak oscillations of the entanglement are clearly visible by eliminating the linear growth, featuring the many-body revivals [19].

By contrast, the fast damping amplitude of fidelity for quench from the $\left|\tilde{\mathbb{Z}}_{4}\right\rangle$ state indicates the negligible overlaps between scarred eigenstates and the $\left|\tilde{\mathbb{Z}}_{4}\right\rangle$ product state. Meanwhile, the entanglement undergoes an extremely fast growth. Note that the bipartite entanglement approaching the maximum entanglement entropy $\log _{2}(\chi d)$ implies a large accumulated error and thus an inevitable breakdown of the density-matrix renormalization group (DMRG) method [46].

Furthermore, it is striking to find that the energy spectra and eigenstates overlap with the Néel state of Eq. (10) also coincide with those of Eq. (8), except the highly degenerate states at $E=0$, implying that a precise relation exists between the eigenstate of spin-1 Kitaev model (8) and the celebrated spin-1/2 PXP model (10). The zero modes of the PXP model 

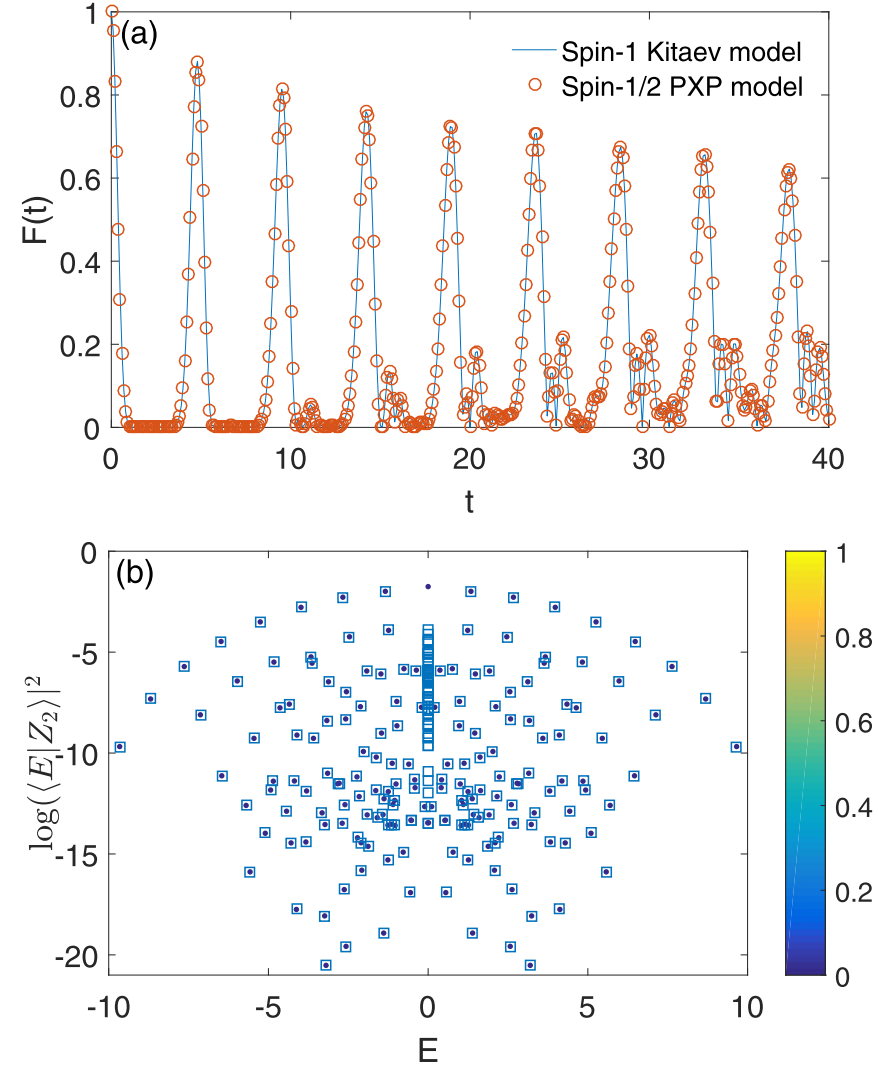

FIG. 2. Characteristic quantum features of the spin-1/2 PXP model. (a) The fidelity $F(t)$ for spin-1 Kitaev model starting from the initial $\left|\tilde{\mathbb{Z}}_{2}\right\rangle\left(\left|\mathbb{Z}_{2}\right\rangle\right)$ states with $N=18$. (b) Eigenstate overlap with the Néel $\mathbb{Z}_{2}$ state for spin-1/2 PXP model (dots) and the $w_{j}=1$ for $(j=1,2, \ldots, N)$ subspace of spin-1 Kitaev model (squares) with $N=16$. Color scale on the right of panel (b) indicates the density of data points, with lighter regions being more dense.
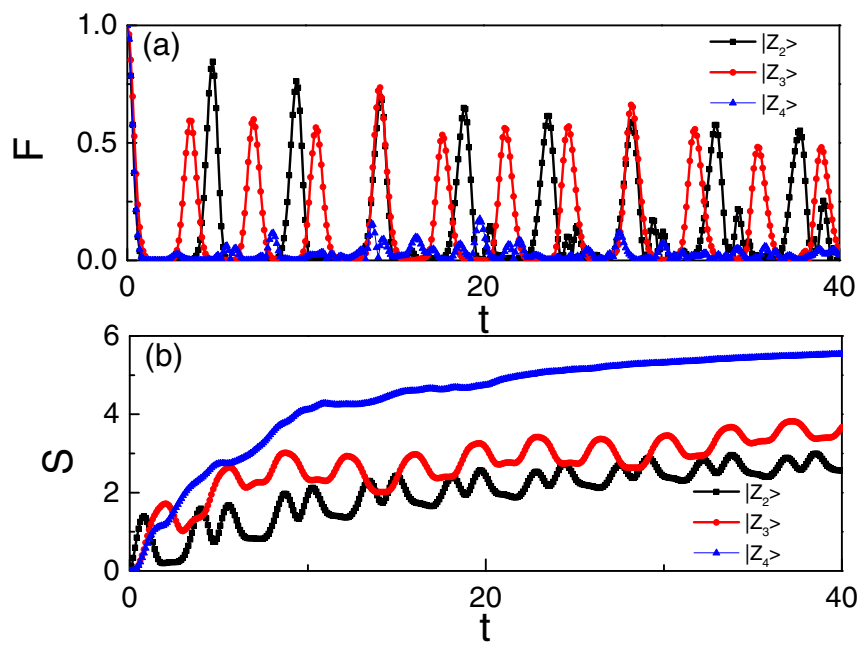

FIG. 3. Quantum features of the 24-site $S=1$ Kitaev chain: (a) the fidelity $F$ and (b) the entanglement $\mathcal{S}$. The results correspond to starting from the initial $\left|\tilde{\mathbb{Z}}_{k}\right\rangle(k=2,3,4)$ states. The bond dimension is set as $\chi=300$.
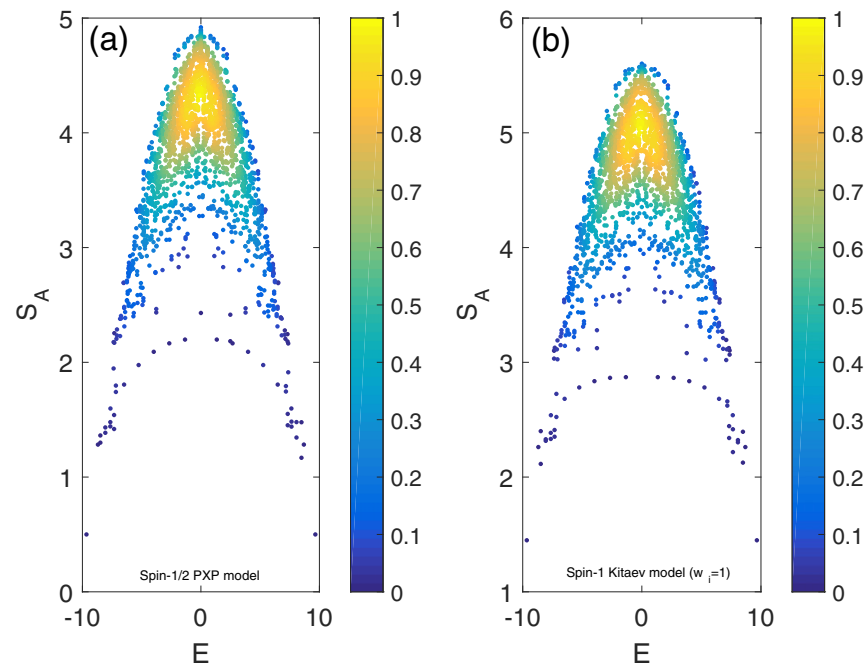

FIG. 4. Bipartite entropy of the eigenstates for (a) spin-1/2 PXP model and (b) spin-1 Kitaev model, within $w_{i}=1(i=1,2, \ldots, N)$ subspace. Color scale indicates the density of data points, with lighter regions being more dense. Here the chain has $N=16$ sites.

at the center of the spectrum are pinned by the particlehole symmetry, i.e., $\left\{\mathcal{C}, H_{\mathrm{PXP}}\right\}=0$, with $\mathcal{C}=\prod_{j=1}^{N} Z_{j}$ [47]. The states with $E=0$ can be classified as eigenstates of $\mathcal{C}$. An exponential growth of protected many-body zero-energy modes with system size arise as a consequence of intertwining of spectral-reflection $\mathcal{C}$ symmetries and relevant point-group symmetries [48].

Among the above macroscopic number of states, two exact scar states for PBC were identified in terms of matrix product states with a finite bond dimension [47], which shows that these exact scar states have constant entanglement. However, the bipartite entropy of the eigenstates of spin- $1 / 2$ PXP model in Fig. 4(a) and the $w_{j}=1(j=1,2, \ldots, N)$ subspace of spin-1 Kitaev model in Fig. 4(b) quantitatively exhibit similar features but are not exactly equivalent. Notably, the different choice of reference bases will modify the values of bipartite entanglement entropy.

The explicit technical insights can be accessible by an exact solution of the two-site model, which are presented in Appendix A. The scarred eigenstates are characterized by the low bipartite entanglement entropy $\mathcal{S}_{A}$, and they become increasingly decoupled from the thermal bulk states as the system size $N$ increases. The primary $\mathbb{Z}_{2}$ scar states can be constructed from the excitations on top of the exact scar states at $E=0$. The equally spaced towers of QMBSs in the spectrum are closely related to perfect revivals, but these characteristics of particular interest are currently limited by small system sizes. However, they are experimentally relevant.

\section{STABILITY OF QMBS AGAINST HEISENBERG INTERACTIONS}

It is appealing to consider the stability of the QMBS to a nonzero perturbation, which could possibly preserve or undermine the conservation of local quantities characteristic of the Kitaev model. Considering atomic states in the $t_{2 g}$ manifold are immersed under the crystalline electric field 

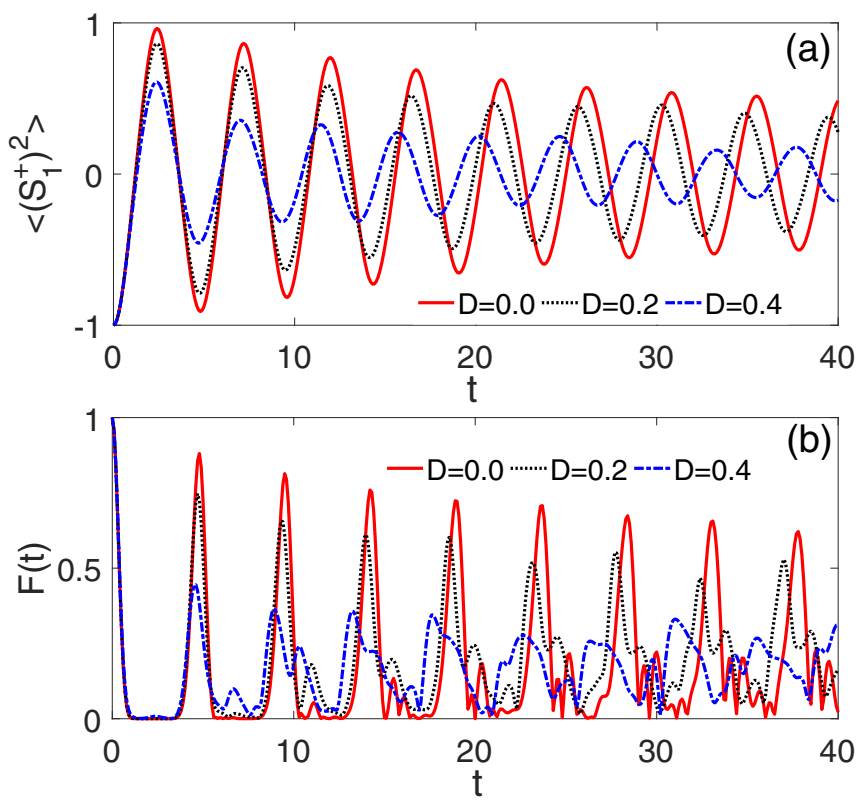

FIG. 5. Time evolution of an 18-site Kitaev chain starting from the initial $\left|\tilde{\mathbb{Z}}_{2}\right\rangle$ state: (a) the order parameter (18) and (b) the quench fidelity (12). The data are presented for increasing strength of singleion anisotropy (17), $D=0.0,0.2,0.4$. See legend.

with the strong spin-orbit coupling, other interactions, such as the Heisenberg exchange, and Dzyaloshinskii-Moriya interaction, coexist with the Kitaev interactions [49]. In addition to the quadratic exchange interactions, the uniaxial single-ion anisotropy (SIA) due to crystal-field effects [50] and furtherneighbor interactions are also ubiquitous [51]. In reality, these interactions play an important role in establishing nontrivial electronic correlations for low-dimensional models with higher spin $S>\frac{1}{2}$.

We are interested in effects induced by perturbations that leave the intermediate symmetries intact. The SIA

$$
\hat{H}_{\mathrm{D}}=D \sum_{j}\left(S_{j}^{z}\right)^{2}
$$

is one of them and leads to a quantum phase transition between a topologically trivial phase and a nontrivial phase in spin-1 AFM Heisenberg chain as predicted by Haldane [52,53]. Simultaneously, the SIA leads to a low-entropy spin Mott insulator in the FM analogy [54,55]. It is easy to find that Eq. (17) is invariant under the rotation (7) and commutes with the local parity operators defined in Eq. (9). We can observe in Fig. 5 that periodic oscillations in the order parameter [56]

$$
O_{1}=\left\langle\left(S_{1}^{+}\right)^{2}\right\rangle
$$

and the quench fidelity persist for long times when starting from $\left|\tilde{\mathbb{Z}}_{2}\right\rangle$. As $D$ increases, the oscillation period gets shorter.

We are also interested in the effects under a perturbation $V \propto\left(S_{j}^{x} S_{j+1}^{z} S_{j+2}^{y}\right)$ that obey the same intermediate $\mathbb{Z}_{2}$ symmetries of Eq. (8). The origin of such three-site $X Z Y$-type interactions is explained in Appendix B. One can observe that the order parameter $O_{1}$ and the quench fidelity $F(t)$ show quite similar oscillations as those of SIA. The state coherence sustains a long time for a moderate perturbation, and the revival will degrade quantitatively with the increasing amplitude of multispin perturbations, while the oscillation period is almost unchanged.

However, it is gradually recognized that isotropic Heisenberg interactions commonly exist in solid-state materials and perturb the systems with frustrated interactions. One frequently considered example is the compass model where Heisenberg interactions are responsible for the onset of possible long-range two-dimensional (2D) order in the ground state, but simultaneously excited states preserve their unique nature which may be used for information storage. Here the nematic order which persists in excited states may be used for correcting the faults along the computations [57,58].

Another example of the robust structure of excited states in the presence of perturbation is encountered in the KitaevHeisenberg model for $S=1 / 2$ spin, which was intensely investigated and several phases with broken symmetry were found $[40,59,60]$. A similar situation was reported as well for the spin-1 Kitaev honeycomb model in candidate materials, such as honeycomb $\mathrm{Ni}$ oxides with heavy elements of $\mathrm{Bi}$ and $\mathrm{Sb}$, where Kitaev interaction is accompanied by a finite FM Heisenberg interaction. In the zero-field limit, the Kitaev QSL is destabilized when $J / K>0.08$ [61]. We thus assume that such interactions are of Heisenberg type

$$
\hat{H}_{\mathrm{J}}=J \sum_{i=1}^{N} \mathbf{S}_{i} \cdot \mathbf{S}_{i+1},
$$

where $J$ stands for the Heisenberg exchange coupling. After the rotation (7), the perturbation by Heisenberg interaction can be rewritten as

$$
\tilde{H}_{\mathrm{J}}=J \sum_{j=1}^{N}\left(S_{j}^{x} S_{j+1}^{y}+S_{j}^{y} S_{j+1}^{x}-S_{j}^{z} S_{j+1}^{z}\right) .
$$

The full Hamiltonian takes an anisotropic translational form

$$
\tilde{H}_{\mathrm{KJ}}=\sum_{j=1}^{N}\left\{(K+J) S_{j}^{x} S_{j+1}^{y}+J S_{j}^{y} S_{j+1}^{x}-J S_{j}^{z} S_{j+1}^{z}\right\} .
$$

A direct consequence is the additional Heisenberg interactions spoil the $\mathbb{Z}_{2}$ symmetry of Eq. (1) associated with each bond. It is worth noting that the energy levels of low-lying excited states cross at $J=0$. The first excited state of $\tilde{H}_{\mathrm{K}}$ is $N$-fold degenerate, corresponding to one $w_{j}=-1$ defect in the sector with all other $w_{j}=1$, such that either $|00+0 \ldots 00\rangle$ or $|00-0 \ldots 00\rangle$ state occurs. A narrow gapped Kitaev phase is capable of sustaining the perturbations of Heisenberg interactions.

The competition between spin-1 AFM Kitaev chain and isotropic Heisenberg interactions was analyzed for $K=1$ [62], while a complete phase diagram, to the best of our knowledge, is elusive and still deserves a careful investigation. To incorporate the phase diagram of the spin-1 KitaevHeisenberg model with $K=1$ [62], the ground-state phase diagram with $K=-1$ is depicted using the DMRG and exact diagonalization methods. In the DMRG simulations, we keep up to $\chi=500$ bond states during the procedure of basis truncation and the number of sweeps is $n=30$. These conditions guarantee that the simulation is converged sufficiently fast and the truncation error is smaller than $10^{-7}$. 

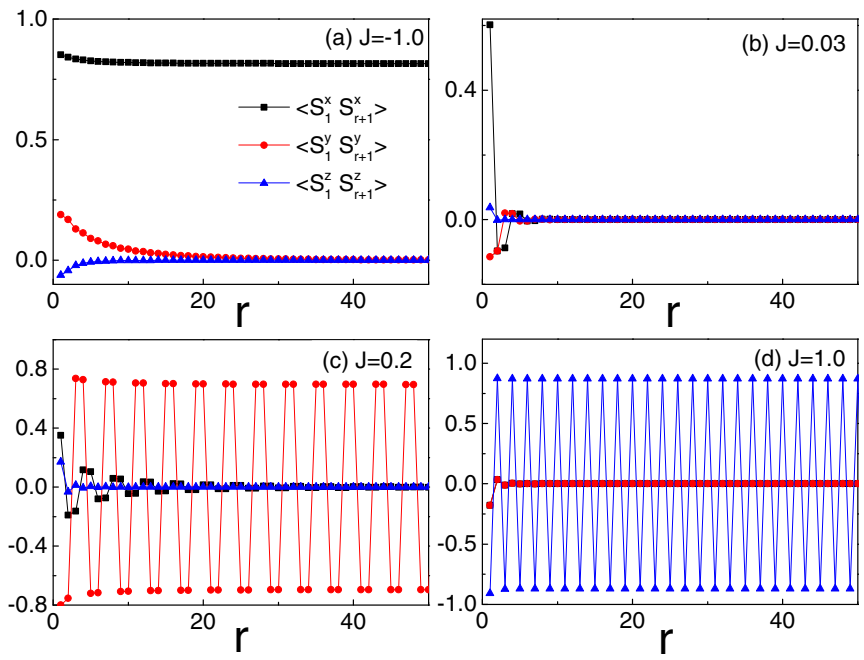

FIG. 6. The correlations between site 1 and $(r+1)$ for increasing distance $r$ and for (a) $J=-1.0$, (b) $J=0.03$, (c) $J=0.2$, and (d) $J=1.0$. Here we use PBCs for the chain with $N=200$ sites.

One motivation for the study of AFM spin-1 Heisenberg chains $(K=0, J>0)$ is that the model hosts a symmetryprotected topological ground state with a finite Haldane gap [63], a reminiscent of AKLT state [64]. It has been known for decades that the indicator of the Haldane phase is beyond any local symmetry-breaking order parameters. Instead, it can be characterized by the exponential decay of two-spin correlation function and nonlocal string order parameters $[65,66]$.

As for the coexistence the Kitaev interaction and the Heisenberg interactions, the spatial inversion symmetry, the time-reversal symmetry, and the dihedral $D_{2}$ symmetry are preserved, which protects the Haldane phase [67,68]. Another specific case occurs with $J \equiv-K / 2$. Then Eq. (21) can be recast into an isotropic form

$$
\tilde{H}_{\mathrm{KJ}}=\frac{1}{2} K \sum_{j=1}^{N}\left(\tilde{S}_{j}^{x} \tilde{S}_{j+1}^{x}+\tilde{S}_{j}^{y} \tilde{S}_{j+1}^{y}+\tilde{S}_{j}^{z} \tilde{S}_{j+1}^{z}\right),
$$

through the following spin rotation:

$$
\begin{aligned}
& \tilde{S}_{j}^{x}=\cos (\pi j / 2) S_{j}^{x}-i \sin (\pi j / 2) S_{j}^{y}, \\
& \tilde{S}_{j}^{y}=\cos (\pi j / 2) S_{j}^{y}+i \sin (\pi j / 2) S_{j}^{x} .
\end{aligned}
$$

In this case, the ground state of Eq. (22) possesses one Goldstone mode, and its character depends on the sign of $K$. The Goldstone mode has a quadratic dispersion for FM Heisenberg model with $K=-1$, while it is gapped for AFM Heisenberg model with $K=1$.

For a generic case, a well-defined order parameter is a vital ingredient for characterizing the nature of phases. To identify the region of the Kitaev phase and the Haldane phase, we calculate the nonlocal correlator

$$
C_{i, j}^{a}=\left\langle S_{i}^{a} \exp \left(i \theta \sum_{l=i+1}^{j-1} S_{l}^{a}\right) S_{j}^{a}\right\rangle, a=x, y, z .
$$

For $\theta=\pi$, Eq. (24) becomes the den NijsRommelse string order parameter whose limiting value $O_{s}^{a}=\lim _{|i-j| \rightarrow \infty}\left(-C_{i, j}^{a}\right)$ reveals the hidden symmetry

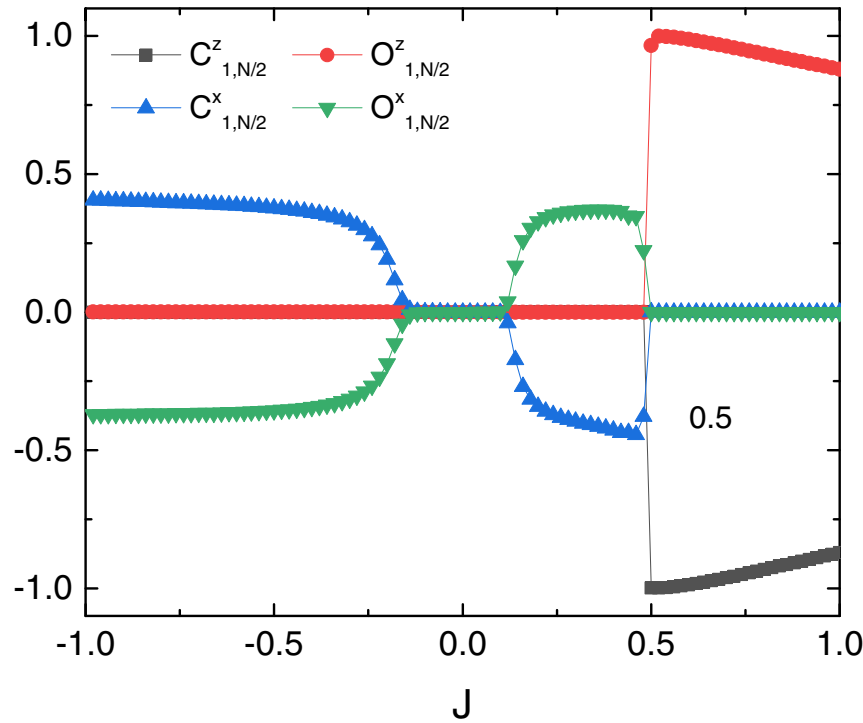

FIG. 7. Two-point correlation $C^{a}(24)$ and string order parameter $O^{a}(a=x, z)$ of 1D pristine spin-1 Kitaev-Heisenberg model for $J \in(-1,1)$ between sites 1 and $N / 2$ for $N=80$. With increasing $J$, the order in the ground state changes from $\mathrm{FM}_{x}$ through QSL and LLRR phase to the Haldane phase.

breaking $[69,70]$. For $\theta=0$, Eq. (24) reduces to two-point correlations.

When $J$ varies for $K=-1$, the competing correlations will trigger miscellaneous phase transitions. For $J \simeq-1$, the $C_{i, j}^{x}$ correlations dominate, suggesting that the ground state is $\mathrm{FM}_{x}$, see Fig. 6(a). For $J \approx 0$, the pure spin- $1 \mathrm{Kitaev}$ chain hosts nearest-neighbor AFM orders due to the $\mathbb{Z}_{2}$ symmetry. The especially short-range correlations are demonstrated for $J=0.03$. The nearest-neighbor spins favor here the FM alignment as the next-nearest-neighbor interactions are FM, this state is shown in Fig. 6(b). When $J$ increases further, the dominating $y$-component correlations have a negative sign on odd bonds and a positive sign on even bonds. These correlations indicate that spin order develops into the left-left-right-right (LLRR) phase, as is shown in Fig. 6(c). Figure 6(d) shows that the $C_{i, j}^{x}$ correlations dominate over $C_{i, j}^{y}$ and $C_{i, j}^{z}$ when $J \simeq 1$.

To clarify the uniaxial order, the two-point correlation $C^{a}$ and the string order parameter $O^{a}(a=x, z)$ between sites 1 and $N / 2$ on a $N=80$ site chain are shown for increasing $J$ in Fig. 7. One can recognize a finite $C_{1, N / 2}^{x}$ that characterizes the $\mathrm{FM}_{x}$ phase for $J<-0.08$, while all correlations vanish in the interval $-0.08<J<0.08$. Next $C_{1, N / 2}^{x}<0$ for $0.08<J<0.50$, while finally $O_{1, N / 2}^{z} \simeq 0.9$ is finite for $J>0.5$ and defines the phase boundary of the Haldane phase.

\section{PHASE DIAGRAM OF THE KITAEV-HEISENBERG CHAIN FOR $S=1$}

The parameters $\{K, J\}$ for the Kitaev and Heisenberg exchange couplings can be set as

$$
K \equiv \sin (\phi), \quad J \equiv \cos (\phi),
$$

where the angle $\phi \in[0,2 \pi)$ parametrizes the Hamiltonian (21). To this end, the complete phase diagram of the $1 \mathrm{D}$ spin-1 


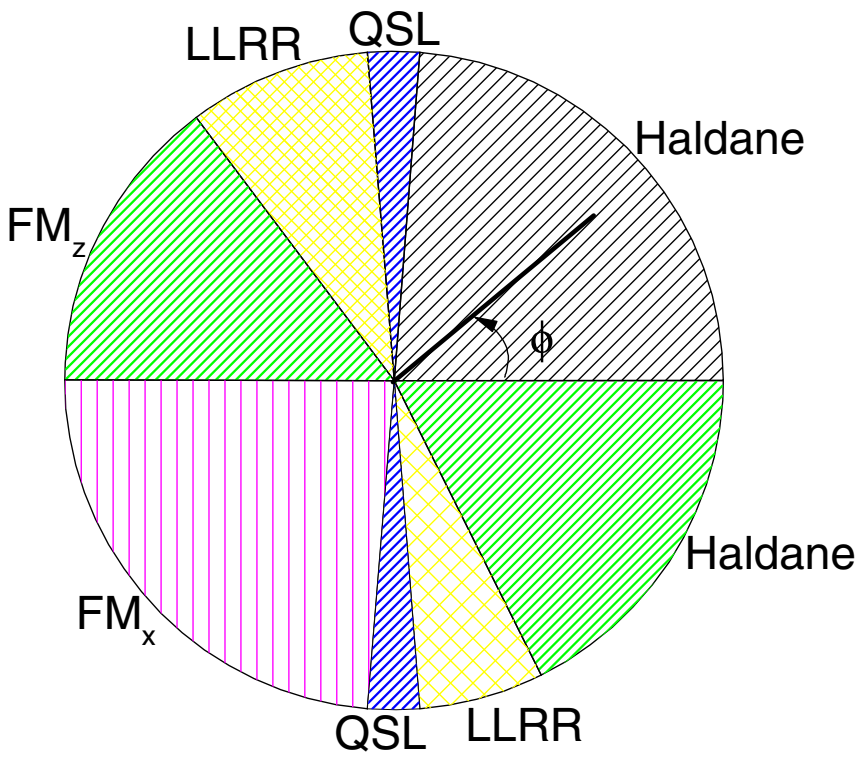

FIG. 8. Ground-state phase diagram of the 1D KitaevHeisenberg model for $S=1$. The vertical (horizontal) axis stands for the Kitaev $K$ (Heisenberg $J$ ) exchange coupling, as given in Eq. (25). Here $\phi \in[0,2 \pi)$ is the angle which determines the exchange constants, see Eq. (25).

Kitaev-Heisenberg model as a function of $\phi$ is displayed in Fig. 8, where the vertical (horizontal) axis is the Kitaev $K$ (Heisenberg $J$ ) exchange coupling. For both cases with either $K>0$ or $K<0$, the phase diagram consists of four different phases, i.e., the FM phase, the Kitaev QSL phase, the LLRR phase, and the Haldane phase. To some extent, the symmetry between the regimes of $K>0$ and $K<0$ is restored.

It is interesting to note that a generalized Kennedy-Tasaki transformation, $U_{\mathrm{KT}}=\prod_{j<k} \exp \left(i \pi S_{j}^{y} S_{k}^{x}\right)[71,72]$, can be realized as the reciprocal transformation between the string order and the local FM order. In this regard, Eqs. (1) and (19) are transformed into equivalent ones with a minus sign standing with short-range interactions, where the nonlocal string observable $O_{i, j}^{a}(\hat{H})$ is responsible for the two-point correlations $C_{i, j}^{a}(\tilde{H})$, with $a=x, z$ of the transformed Hamiltonian

$$
\begin{aligned}
\tilde{H}_{\mathrm{KJ}}^{\prime}= & -K \sum_{j=1}^{N / 2}\left(S_{2 j-1}^{x} S_{2 j}^{x}+S_{2 j}^{y} S_{2 j+1}^{y}\right) \\
& -J \sum_{j=1}^{N}\left(S_{j}^{x} S_{j+1}^{x}+S_{j}^{y} S_{j+1}^{y}+\tilde{W}_{j} S_{j}^{z} S_{j+1}^{z}\right) .
\end{aligned}
$$

This suggests a likely relationship $\tilde{H}(-K,-J) \simeq \hat{H}(K, J)$.

Notably, the coupled $\mathbb{Z}_{2}$ gauge fields $\left\{\tilde{W}_{j}\right\}$ are not conserved in the transformed pristine Hamiltonian. The duality becomes exact when the strength of the Heisenberg interaction $J$ is tiny $(\phi \approx \pm \pi / 2)$, which means that the parameters are within the boundaries of the Kitaev QSL phases. However, Fig. 8 clearly shows that such a relationship does not hold for moderate values of $J$ owing to quantum fluctuations of Ising gauge fields. The system undergoes a second-order quantum phase transition from the FM phase to the LLRR phase at $\phi=0.8280 \pi$ (i.e., for $K=1, J=-0.6$ ), while another tran-
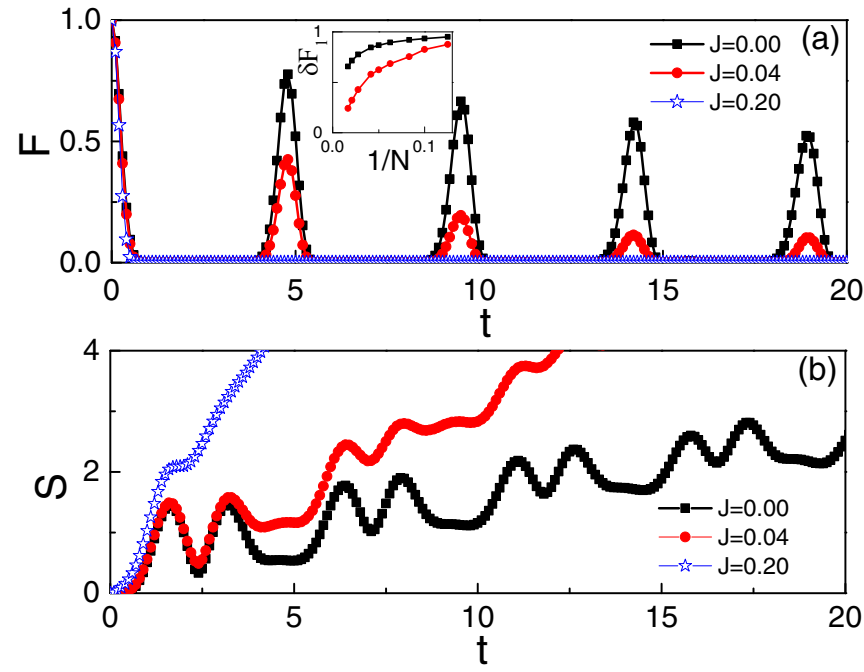

FIG. 9. Quantum characteristics of the $S=1 \mathrm{Kitaev-Heisenberg}$ chain of $N=36$ sites, starting from $|-+-+\cdots-+\rangle$ in Eq. (21) as the initial state for selected values of $J$ : (a) the fidelity $F$ and (b) the entanglement entropy $\mathcal{S}$. The inset in (a) shows the scaling of the first revival peak $\delta F_{1}$ versus $1 / N$ for $J=0.0$ and $J=0.04$. The bond dimension is set as $\chi=400$.

sition from the LLRR phase to the Haldane phase occurs at $\phi=1.8524 \pi$ (i.e., for $K=-1, J=0.5$ ). In fact, there is no phase transition at $\phi=0(K=0, J=1)$, where the string observables $O_{i, j}^{a}$ are balanced in both the $x$ - and $z$-directions, corresponding to the restoration of hidden $\mathbb{Z}_{2} \times \mathbb{Z}_{2}$ symmetry breaking.

We note that the phase diagram of the $S=1$ KitaevHeisenberg chain resembles to some extent the phase diagram of the Kitaev-Heisenberg chain for $S=\frac{1}{2}$ spin [60], and one finds the following correspondence between phases: the Haldane phase for $S=1$ corresponds to the AFM phase for $S=\frac{1}{2}$ and the FM phase for $S=1$ corresponds again to the FM phase when spin is smaller. But it is remarkable that the LLRR phase corresponds to two distinct phases when $S=\frac{1}{2}$ : the zigzag phase for $K>0$ and the stripe phase for $K<0$. Thereby the regime of the LLRR phase for $K>0$ extends over a broader range of angle $\phi$, similar to the broader range of $\phi$ for the zigzag phase at $S=\frac{1}{2}$ [60]. In addition, both the FM and the Haldane phase consist here of two distinct phases, depending on whether $K>0$ or $K<0$. This demonstrates that the quantum components of $S=1$ spins are nonequivalent and the SU(2) symmetry of the Heisenberg term does not hold when Kitaev interactions are finite.

Finally, we investigate the scar stability in a broader regime, which is relevant for possible solid-state applications. Then we consider the scar stability of the Kitaev model with Heisenberg perturbations, and show that they display anomalous stability in the Kitaev phase. Figure 9 shows the fidelity with periodic slowly decaying revivals in the dynamics for $J=0$. In contrast, it completely collapses for $J=0.2$ with $N=36$. The coherent dynamics is retained in the Kitaev phase while it is lost quickly when the phase enters other phases. Away from the Kitaev point, one can observe how the entanglement grows rapidly with time. These results suggest that exact scars are a generic property of Kitaev models. 


\section{SUMMARY AND CONCLUSION}

In this paper, we studied the eigenstate properties of Hamiltonian relevant for spin-orbit-coupled electronic system, where the PXP model was embedded. Thus, the many-body quantum scars and the associated constrained dynamics can be unveiled. This phenomenology is characterized by the fact that the dynamics is anomalously slow provided the initial state has a nonnegligible overlap with scarred states. The ETH violations were explicitly detected in the uniform $w_{j}=1(\forall j)$ sector, where the spin-1 Kitaev model can be rigorously mapped into a spin- $\frac{1}{2}$ PXP model. The PXP model acts here as an archetypal model to possess quantum scars, which are introduced to explain the slow dynamics observed by evolving a charge-density wave initial state in the Rydberg atom chain realized in Ref. [20]. As a consequence of nearest-neighbor Rydberg blockaded, the dimension of constrained Hilbert space grows as $g^{N}$, where $g=(1+\sqrt{5}) / 2$ is the golden ratio. A few scarred eigenstates spread throughout the spectrum and their presence is evidenced by subextensive entanglement entropy and persistent oscillation of local observables. We showed that there is the coexistence of volumelaw and area-law entangled eigenstates throughout the spectrum.

These ETH violating eigenstates survive despite hybridization with an exponential number of thermal eigenstates and motivated the study of their stability against perturbation. We consider the perturbations by Heisenberg interactions, see Eq. (26). To characterize the ground-state properties, we adopt both the local and nonlocal correlations, which identify distinct phases when the Kitaev coupling $K$ and the Heisenberg coupling $J$ vary, see Fig. 8. For large negative $J$, the FM order along the $z(x)$ axis is favored for $K=1(K=-1)$. Increasing the value of $J$, the ground state evolves from the FM state into the stripy phase, in which the spin structure has a left-left-right-right pattern. The magnetic order vanishes when $J$ approaches the Kitaev limit. In stark contrast to the gapless ground state of the $S=\frac{1}{2}$ Kitaev chain, the gapped ground state supports a narrow Kitaev phase in the vicinity of $J=0$, which is characterized by the extremely short-range correlations.

As a universal feature, the system enters into the Haldane phase upon increasing $J$. The Haldane phase maintains its topological character and cannot evolve adiabatically to other phases since it is protected by the combination of the spatial inversion symmetry, the time-reversal symmetry, and the dihedral $D_{2}$ symmetry. The continuous revivals for starting from particular initial states remains in the disordered Kitaev phase, while the state coherence breaks down swiftly in the symmetry-breaking phases. Nevertheless, with the help of examples of the single-ion term and multispin interactions, we demonstrate the scarred states appear to be robust against $\mathbb{Z}_{2}$-symmetry preserving perturbations, at least for the currently experimentally accessible system-sizes. Our findings highlight that spin-1 Kitaev systems exhibit a rich variety of phenomena and thus provide a generic nonintegrable constrained quantum many-body system to study nonergodic behaviors, the stability under generic perturbations, and quantum analogs of the Kolmogorov-Arnold-Moser theorem.

\section{ACKNOWLEDGMENTS}

The authors appreciate very insightful discussions with Wojciech Brzezicki, Hosho Katsura, and Zhi-Xiang Sun. This work is supported by the National Natural Science Foundation of China (NSFC) under Grant No. 12174194, the startup fund of Nanjing University of Aeronautics and Astronautics Grant No. 1008-YAH20006, Top-notch Academic Programs Project of Jiangsu Higher Education Institutions (TAPP) and stable supports for basic institute research, Grant No. 190101. A. M. Oleś kindly acknowledges support by Narodowe Centrum Nauki (NCN, Poland) under Project No. 2016/23/B/ST3/00839 and is grateful for support via the Alexander von Humboldt Foundation Fellowship [73] (Humboldt-Forschungspreis).

\section{APPENDIX A: BIPARTITE ENTROPY OF TWO-SITE SPIN-1/2 PXP MODEL AND SPIN-1 KITAEV MODEL}

Here we consider $N=2$. The Hamiltonian of the spin- $1 / 2$ PXP model within the bases in the constrained Hilbert space

$$
\begin{aligned}
& \{|\downarrow \downarrow\rangle,|\downarrow \uparrow\rangle,|\uparrow \downarrow\rangle\}, \\
& H=\left(\begin{array}{lll}
0 & 1 & 1 \\
1 & 0 & 0 \\
1 & 0 & 0
\end{array}\right),
\end{aligned}
$$

and the ones for the spin-1 Kitaev model within $\left(w_{i}=1\right.$, $i=1,2, \ldots, N)$ subspace

$$
\begin{gathered}
\{|-+\rangle,|+-\rangle,|00\rangle\}, \\
H=\left(\begin{array}{lll}
0 & 0 & 1 \\
0 & 0 & 1 \\
1 & 1 & 0
\end{array}\right) .
\end{gathered}
$$

There is a one-to-one correspondence between Eqs. (A1) and (A3):

$$
|-+\rangle \Rightarrow|\downarrow \uparrow\rangle,|+-\rangle \Rightarrow|\uparrow \downarrow\rangle,|00\rangle \Rightarrow|\downarrow \downarrow\rangle .
$$

The eigenvectors of Eq. (A2)

$$
\begin{aligned}
& \left|\psi_{1}\right\rangle=-0.7071|\downarrow \downarrow\rangle+0.5000|\downarrow \uparrow\rangle+0.5000|\uparrow \downarrow\rangle, \\
& \left|\psi_{2}\right\rangle=0.0000|\downarrow \downarrow\rangle-0.7071|\downarrow \uparrow\rangle+0.7071|\uparrow \downarrow\rangle, \\
& \left|\psi_{3}\right\rangle=0.7071|\downarrow \downarrow\rangle+0.5000|\downarrow \uparrow\rangle+0.5000|\uparrow \downarrow\rangle .
\end{aligned}
$$

The entanglement spectra and von Neumann entropy

$$
\begin{array}{cc}
\lambda_{1,3}^{(1,2)}=\frac{1}{2} \pm \frac{\sqrt{3}}{4}, & \text { with } \quad S_{A}=0.3546, \\
\lambda_{2}^{(1,2)}=\frac{1}{2}, \frac{1}{2}, \quad \text { with } \quad S_{A}=1.0 .
\end{array}
$$

Similarly, the eigenvectors of Eq. (A4)

$$
\begin{aligned}
& \left|\phi_{1}\right\rangle=0.5000|-+\rangle+0.5000|+-\rangle-0.7071|00\rangle, \\
& \left|\phi_{2}\right\rangle=0.7071|-+\rangle-0.7071|+-\rangle+0.0000|00\rangle, \\
& \left|\phi_{3}\right\rangle=0.5000|-+\rangle+0.5000|+-\rangle+0.7071|00\rangle .
\end{aligned}
$$


The entanglement spectra and von Neumann entropy are

$$
\begin{aligned}
& \lambda_{1,3}^{(1,2,3)}=\frac{1}{4}, \frac{1}{4}, \frac{1}{2}, \quad \text { with } \quad S_{A}=1.5, \\
& \lambda_{2}^{(1,2,3)}=\frac{1}{2}, \frac{1}{2}, 0, \quad \text { with } \quad S_{A}=1.0 .
\end{aligned}
$$

To this end, the diverse choice of bases in Eqs. (A1) and (A3) will lead to multiple values of bipartite entanglement entropy.

\section{APPENDIX B: KITAEV CHAIN UNDER $X Z Y$-TYPE PERTURBATIONS}

The energy current follows from the continuity equation [74]

$$
\hat{J}_{\mathrm{E}}=\sum_{j=1}^{N} i\left[h_{j}, h_{j+1}\right]=K^{2} \sum_{j=1}^{N} S_{j}^{x} S_{j+1}^{z} S_{j+2}^{y} .
$$

This operator acts on three adjacent sites and contains also the $z$ component of spin-1 operators, which commutes with $\tilde{W}_{j}$. The presence of an effective energy flow will manifest itself in the effective Hamiltonian followed by a Lagrange multiplier $\lambda$ :

$$
\tilde{H}_{\mathrm{K}}=K \sum_{j=1}^{N} S_{j}^{x} S_{j+1}^{y}-\lambda \hat{J}_{\mathrm{E}}
$$

It can be verified that $\left[\tilde{W}_{j}, \tilde{W}_{k}\right]=0,\left[\tilde{W}_{j}, \tilde{H}_{\mathrm{K}}\right]=0$. In this respect, the three-site $X Z Y$-type interactions belong to
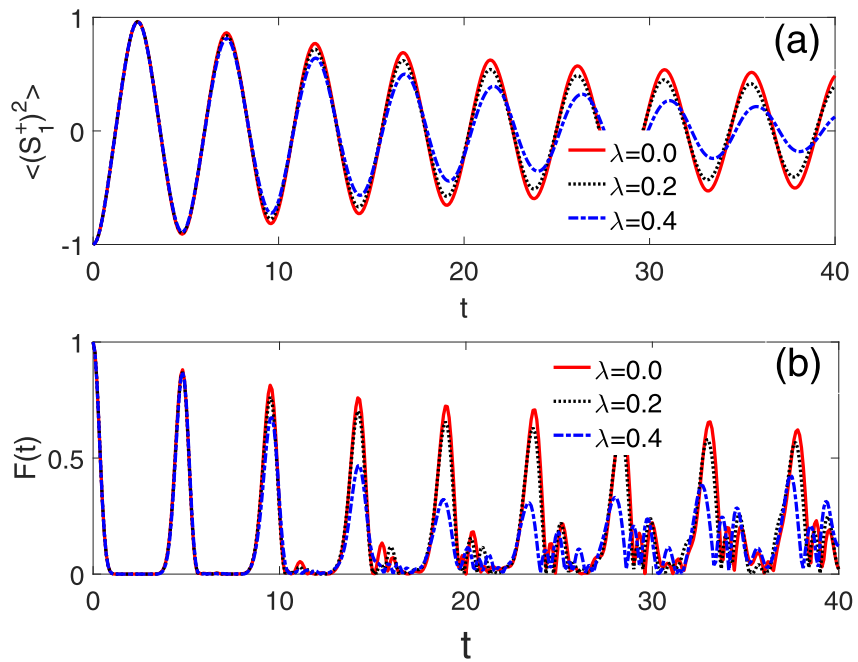

FIG. 10. Quantum properties of the Kitaev chain with $N=18$ sites starting from the initial $\left|\tilde{\mathbb{Z}}_{2}\right\rangle$ state with $X Z Y$ interactions and for $\lambda=0.0,0.2,0.4$ : (a) the order parameter (18) and (b) the quench fidelity $F(t)$.

$\mathbb{Z}_{2}$-symmetry preserved perturbations. The numerical results for different values of $\lambda$ are shown in Fig. 10. One finds that the oscillations of the order parameter (18) are more damped and the fidelity $F(t)$ has a richer structure at larger time $t$ when $\lambda$ increases.
[1] D. A. Abanin, E. Altman, I. Bloch, and M. Serbyn, Colloquium: Many-body localization, thermalization, and entanglement, Rev. Mod. Phys. 91, 021001 (2019).

[2] M. Rigol, V. Dunjko, V. Yurovsky, and M. Olshanii, Relaxation in a Completely Integrable Many-Body Quantum System: An $A b$ Initio Study of the Dynamics of the Highly Excited States of 1D Lattice Hard-Core Bosons, Phys. Rev. Lett. 98, 050405 (2007).

[3] M. Rigol, V. Dunjko, and M. Olshanii, Thermalization and its mechanism for generic isolated quantum systems, Nature (London) 452, 854 (2008).

[4] A. Polkovnikov, K. Sengupta, A. Silva, and M. Vengalattore, Colloquium: Nonequilibrium dynamics of closed interacting quantum systems, Rev. Mod. Phys. 83, 863 (2011).

[5] J. Sirker, A. Herzog, A. M. Oleś, and P. Horsch, Thermally Activated Peierls Dimerization in Ferromagnetic Spin Chains, Phys. Rev. Lett. 101, 157204 (2008).

[6] P. W. Anderson, Absence of diffusion in certain random lattices, Phys. Rev. 109, 1492 (1958).

[7] M. Brenes, T. Leblond, J. Goold, and M. Rigol, Eigenstate Thermalization in a Locally Perturbed Integrable System, Phys. Rev. Lett. 125, 070605 (2020).

[8] D. M. Basko, I. L. Aleiner, and B. L. Altshuler, Metal-insulator transition in a weakly interacting many-electron system with localized single-particle states, Ann. Phys. (NY) 321, 1126 (2006).
[9] A. Pal and D. Huse, The many-body localization phase transition, Phys. Rev. B 82, 174411 (2010).

[10] M. Žnidarič, T. Prosen, and P. Prelovšek, Many body localization in Heisenberg XXZ magnet in a random field, Phys. Rev. B 77, 064426 (2008)

[11] J. H. Bardarson, F. Pollmann, and J. E. Moore, Unbounded Growth of Entanglement in Models of Many-Body Localization, Phys. Rev. Lett. 109, 017202 (2012).

[12] B. Mukherjee, Z. Cai, and W. Vincent Liu, Constraint-induced breaking and restoration of ergodicity in spin-1 PXP models, Phys. Rev. Research 3, 033201 (2021).

[13] J. Z. Imbrie, On many-body localization for quantum spin chains, J. Stat. Phys. 163, 998 (2016).

[14] Q. Guo, C. Cheng, Z.-H. Sun, Z. Song, H. Li, Z. Wang, W. Ren, H. Dong, D. Zheng, Y.-R. Zhang, R. Mondaini, H. Fan, and $\mathrm{H}$. Wang, Observation of energy-resolved many-body localization, Nat. Phys. 17, 234 (2021).

[15] F. M. Surace, M. Votto, E. G. Lazo, A. Silva, M. Dalmonte, and G. Giudici, Exact many-body scars and their stability in constrained quantum chains, Phys. Rev. B 103, 104302 (2021).

[16] P. Fendley, K. Sengupta, and S. Sachdev, Competing densitywave orders in a one-dimensional hard-boson model, Phys. Rev. B 69, 075106 (2004).

[17] I. Lesanovsky, Liquid Ground State, Gap, and Excited States of a Strongly Correlated Spin Chain, Phys. Rev. Lett. 108, 105301 (2012). 
[18] C. J. Turner, A. A. Michailidis, D. A. Abanin, M. Serbyn, and Z. Papić, Quantum scarred eigenstates in a Rydberg atom chain: Entanglement, breakdown of thermalization, and stability to perturbations, Phys. Rev. B 98, 155134 (2018).

[19] C. J. Turner, A. A. Michailidis, and D. A. Abanin, Weak ergodicity breaking from quantum many-body scars, Nat. Phys. 14, 745 (2018).

[20] H. Bernien, S. Schwartz, A. Keesling, H. Levine, A. Omran, H. Pichler, S. Choi, A. S. Zibrov, M. Endres, M. Greiner, V. Vuletić, and M. D. Lukin, Probing many-body dynamics on a 51-atom quantum simulator, Nature (London) 551, 579 (2017).

[21] W. Kao, K.-Y. Li, K.-Y. Lin, S. Gophalakrishnan, and L. B. Lev, Topological pumping of a 1d dipolar gas into strongly correlated prethermal states, Science 371, 296 (2021).

[22] E. J. Heller, Bound-State Eigenfunctions of Classically Chaotic Hamiltonian Systems: Scars of Periodic Orbits, Phys. Rev. Lett. 53, 1515 (1984).

[23] K. Pakrouski, P. N. Pallegar, F. K. Popov, and I. R. Klebanov, Many-Body Scars as a Group Invariant Sector of Hilbert Space, Phys. Rev. Lett. 125, 230602 (2020).

[24] J. Ren, C. Liang, and C. Fang, Quasisymmetry Groups and Many-Body Scar Dynamics, Phys. Rev. Lett. 126, 120604 (2021).

[25] V. Khemani, C. R. Laumann, and A. Chandran, Signatures of integrability in the dynamics of Rydberg-blockaded chains, Phys. Rev. B 99, 161101(R) (2019).

[26] B. Buča, J. Tindall, and D. Jaksch, Non-stationary coherent quantum many-body dynamics through dissipation, Nat. Commun. 10, 1730 (2019).

[27] S. Choi, C. J. Turner, H. Pichler, and W. W. Ho, Emergent SU(2) Dynamics and Perfect Quantum Many-Body Scars, Phys. Rev. Lett. 122, 220603 (2019).

[28] T. Iadecola and M. Schecter, Quantum many-body scar states with emergent kinetic constraints and finite-entanglement revivals, Phys. Rev. B 101, 024306 (2020).

[29] S. Chattopadhyay, H. Pichler, M. D. Lukin, and W. H. Wen, Quantum many-body scars from virtual entangled pairs, Phys. Rev. B 101, 174308 (2020).

[30] S. Moudgalya, S. Rachel, B. A. Bernevig, and N. Regnault, Exact excited states of non-integrable models, Phys. Rev. B 98, 235155 (2018).

[31] S. Moudgalya, N. Regnault, and B. A. Bernevig, Entanglement of exact excited states of Affleck-Kennedy-Lieb-Tasaki models Exact results, many-body scars, and violation of the strong eigenstate thermalization hypothesis, Phys. Rev. B 98, 235156 (1998).

[32] D. K. Mark, C. J. Lin, and O. I. Motrunich, Unified structure for exact towers of scar states in the AKLT and other models, Phys. Rev. B 101, 195131 (2020).

[33] S. Moudgalya, N. Regnault, and B. A. Bernevig, $\eta$-pairing in Hubbard models: From spectrum generating algebras to quantum many-body scars, Phys. Rev. B 102, 085140 (2020).

[34] D. K. Mark and O. I. Motrunich, $\eta$-pairing states as true scars in an extended Hubbard model, Phys. Rev. B 102, 075132 (2020).

[35] J.-Y. Desaules, A. Hudomal, C. J. Turner, and Z. Papić, Proposal for Realizing Quantum Scars in the Tilted 1D Fermi-Hubbard Model, Phys. Rev. Lett. 126, 210601 (2021).

[36] Z. Nussinov and J. van den Brink, Compass models: Theory and physical motivations, Rev. Mod. Phys. 87, 1 (2015).
[37] A. Y. Kitaev, Anyons in an exactly solved model and beyond, Ann. Phys. (Amsterdam) 321, 2 (2006).

[38] G. Jackeli and G. Khaliullin, Mott Insulators in the Strong Spin-Orbit Coupling Limit: From Heisenberg to a Quantum Compass and Kitaev Models, Phys. Rev. Lett. 102, 017205 (2009).

[39] P. P. Stavropoulos, D. Pereira, and H.-Y. Kee, Microscopic Mechanism for a Higher-Spin Kitaev Model, Phys. Rev. Lett. 123, 037203 (2019).

[40] S. M. Winter, A. A. Tsirlin, M. Daghofer, J. van den Brink, Y. Singh, P. Gegenwart, and R. Valentí, Models and materials for generalized Kitaev magnetism, J. Phys.: Condensed Matter 29, 493002 (2017).

[41] J. A. Sears, L. E. Chern, S. Kim, P. J. Bereciartua, S. Francoual, Y. B. Kim, and Y. J. Kim, Ferromagnetic Kitaev interaction and the origin of large magnetic anisotropy in $\alpha-\mathrm{RuCl}_{3}$, Nat. Phys. 16, 837 (2020).

[42] W.-L. You and G.-S. Tian, Quantum physics transition in the one-dimensionsal compass model using the pseudospin approach, Phys. Rev. B 78, 184406 (2008).

[43] D. Sen, R. Shankar, D. Dhar, and K. Ramola, Spin-1 Kitaev model in one dimension, Phys. Rev. B 82, 195435 (2010).

[44] B. Sun and F. Robicheaux, Numerical study of two-body correlation in a 1D lattice with perfect blockade, New. J. Phys. 10, 045032 (2008).

[45] M. Serbyn, D. A. Abanin, and Z. Papić, Quantum many-body scars and weak breaking of ergodicity, Nat. Phys. 17, 675 (2021).

[46] D. Gobert, C. Kollath, U. Schollwöck, and G. Schütz, Realtime dynamics in spin- $1 / 2$ chains with adaptive time-dependent density matrix renormalization group, Phys. Rev. E 71, 036102 (2005).

[47] C.-J. Lin and O. I. Motrunich, Exact Quantum Many-Body Scar States in the Rydberg-Blockaded Atom Chain, Phys. Rev. Lett. 122, 173401 (2019).

[48] M. Schecter and T. Iadecola, Many-body spectral reflection symmetry and protected infinite-temperature degeneracy, Phys. Rev. B 98, 035139 (2018).

[49] R. Sano, Y. Kato, and Y. Motome, Kitaev-Heisenberg Hamiltonian for High-Spin $d^{7}$ Mott Insulators, Phys. Rev. B 97, 014408 (2018).

[50] C. Xu, J. Feng, H. Xiang, and L. Bellaiche, Interplay between Kitaev interaction and single ion anisotropy in ferromagnetic $\mathrm{CrI}_{3}$ and $\mathrm{CrGeTe}_{3}$ monolayers, npj Comput. Mater. 4, 57 (2018).

[51] D. Pesin and L. Balents, Mott physics and band topology in materials with strong spin-orbit interaction, Nat. Phys. 6, 376 (2010).

[52] F. D. M. Haldane, Continuum dynamics of the 1D Heisenberg antiferromagnet: Identification with the $\mathrm{O}(3)$ nonlinear Sigma model, Phys. Lett. A 93, 464 (1983).

[53] F. D. M. Haldane, Nonlinear Field Theory of Large-Spin Heisenberg Antiferromagnets: Semiclassically Quantized Solitons of the One-Dimensional Easy-Axis Néel State, Phys. Rev. Lett. 50, 1153 (1983).

[54] W. C. Chung, J. de Hond, J. Xiang, E. Cruz-Colón, and W. Ketterle, Tunable Single-Ion Anisotropy in Spin-1 Models Realized with Ultracold Atoms, Phys. Rev. Lett. 126, 163203 (2021). 
[55] E. Altman, W. Hofstetter, E. Demler, and M. D. Lukin, Phase diagram of two-component bosons on an optical lattice, New J. Phys. 5, 113 (2003).

[56] M. Schecter and T. Iadecola, Weak Ergodicity Breaking and Quantum Many-Body Scars in Spin-1 XY Magnets, Phys. Rev. Lett. 123, 147201 (2019).

[57] F. Trousselet, A. M. Oleś, and P. Horsch, Magnetic properties of nanoscale compass-Heisenberg planar clusters, Phys. Rev. B 86, 134412 (2012).

[58] F. Trousselet, A. M. Oleś, and P. Horsch, Compass-Heisenberg model on the square lattice-spin order and elementary excitations, Europhys. Lett. 91, 40005 (2010).

[59] J. Chaloupka, G. Jackeli, and G. Khaliullin, Kitaev-Heisenberg Model on Honeycomb Lattice: Possible Exotic Phases in Iridium Oxides $A_{2} \mathrm{IrO}_{3}$, Phys. Rev. Lett. 105, 027204 (2010).

[60] D. Gotfryd, J. Rusnačko, K. Wohlfeld, G. Jackeli, J. Chaloupka, and A. M. Oleś, Phase diagram and spin correlations of the Kitaev-Heisenberg model: Importance of quantum effects, Phys. Rev. B 95, 024426 (2017).

[61] C. Hickey, C. Berke, P. P. Stavropoulos, H.-Y. Kee, and S. Trebst, Field-driven gapless spin liquid in the spin-1 Kitaev honeycomb model, Phys. Rev. Research 2, 023361 (2020).

[62] W.-L. You, G. Sun, J. Ren, W. C. Yu, and A. M. Oleś, Quantum phase transitions in the spin-1 Kitaev-Heisenberg chain, Phys. Rev. B 102, 144437 (2020).

[63] S. R. White and D. A. Huse, Numerical renormalization group study of low-lying eigenstates of the antiferromagnetic $S=1$ Heisenberg chain, Phys. Rev. B 48, 3844 (1993).
[64] I. Affleck, T. Kennedy, E. H. Lieb, and H. Tasaki, Rigorous Results on Valence-Bond Ground States in Antiferromagnets, Phys. Rev. Lett. 59, 799 (1987).

[65] M. den Nijs and K. Rommelse, Preroughening transitions in crystal surfaces and valence-bond phases in quantum spin chains, Phys. Rev. B 40, 4709 (1989).

[66] H. Tasaki, Quantum Liquid in Antiferromagnetic Chains: A Stochastic Geometric Approach to the Haldane Gap, Phys. Rev. Lett. 66, 798 (1991).

[67] F. Pollmann, A. M. Turner, E. Berg, and M. Oshikawa, Entanglement spectrum of a topological phase in one dimension, Phys. Rev. B 81, 064439 (2010).

[68] F. Pollmann, E. Berg, A. M. Turner, and M. Oshikawa, Symmetry protection of topological phases in one-dimensional quantum spin systems, Phys. Rev. B 85, 075125 (2012).

[69] T. Kennedy and H. Tasaki, Hidden $\mathbb{Z}_{2} \times \mathbb{Z}_{2}$ symmetry breaking in Haldane-gap antiferromagnets, Phys. Rev. B 45, 304 (1992).

[70] S. Takada and K. Kubo, Nonlocal unitary transformations on $S=1$ antiferromagnetic spin chains, J. Phys. Soc. Jpn. 60, 4026 (1991).

[71] T. Kennedy and H. Tasaki, Hidden symmetry breaking and the Haldane phase in $S=1$ quantum spin chains, Commun. Math. Phys. 147, 431 (1992).

[72] M. Oshikawa, Hidden $\mathbb{Z}_{2} \times \mathbb{Z}_{2}$ symmetry in quantum spin chains with arbitrary integer spin, J. Phys.: Condens. Matter 4, 7469 (1992).

[73] https://www.humboldt-foundation.de/web/humboldtpreis.html.

[74] X. Zotos, F. Naef, and P. Prelovšek, Transport and conservation laws, Phys. Rev. B 55, 11029 (1997). 